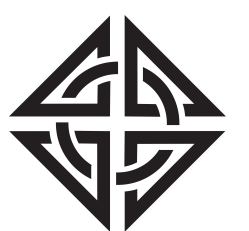

SCIENTIA
Sharif University of Technology

Scientia Iranica

Transactions F: Nanotechnology

http://scientiairanica.sharif.edu

\title{
Multi-scale simulation of SU8 and SU8-graphene nanocomposites: Bridging atomistic to macroscale peridynamics
}

\author{
F. Mohammadzadeh Honarvar ${ }^{a}$, B. Pourabbas ${ }^{a, *}$, M. Salami Hosseini ${ }^{a}$, \\ M. Kharazi ${ }^{\mathrm{b}}$, and H. Erfan-Niya ${ }^{\mathrm{c}}$ \\ a. Department of Polymer Engineering, Nanostructured Materials Research Centre, Sahand University of Technology, Sahand New \\ Town, Tabriz, Iran. \\ b. Department of Mechanical Engineering, Sahand University of Technology, Sahand New Town, Tabriz, Iran. \\ c. Department of Chemical and Petroleum Engineering, University of Tabriz, Tabriz, Iran.
}

Received 27 April 2018; received in revised form 16 November 2018; accepted 26 January 2019

\author{
KEYWORDS \\ Multi-scale; \\ Molecular dynamics; \\ Peridynamics; \\ Simulation; \\ SU8; \\ Graphene; \\ Nanocomposite.
}

\begin{abstract}
SU8 is a commercial epoxy-Novolac resin, a negative-tone photoresist with outstanding mechanical properties. Its nanocomposites have also been considered as research materials. In order to obtain insights into the SU8 nanocomposites with graphene, the present work was conducted to simulate the mechanical properties using multi-scale simulation method including atomistic, meso, and macro scales. The study started by molecular dynamics, then moved to coarse grain, and finally reached macroscale. The methodology applied throughout the work was Peridynamics. Top-down and bottom-up loops were required to confirm the total results. Tensile deformation was applied to a $2 \mathrm{D}$ plane at the upmost scale to create an internal pressure. It was transferred to the lower scale in the next step as the external pressure. The procedure continued down until the molecular scale was reached. However, bottom-up strategy required a bridging model to link molecular scale to the upper scales. The check points were the deformation values, which had to be in the same order independent of top-down or bottom-up movement. With $2.1 \mathrm{wt} . \%$ graphene in SU8, the increases in Young's, bulk, and shear moduli were calculated $(62,200$, and $82 \%$, respectively) compared to the neat SU8.

(C) 2019 Sharif University of Technology. All rights reserved.
\end{abstract}

\section{Introduction}

Polymeric nanocomposites are polymers consisting in nanoscale materials such as nanotubes, nano-spheres,

\footnotetext{
*. Corresponding author. Tel.: +984133449083;

Fax: +984133444303

E-mail addresses: f_mohamadzadeh@sut.ac.ir (F.

Mohammadzadeh Honarvar); pourabas@sut.ac.ir (B.

Pourabbas); salami@sut.ac.ir (M. Salami Hosseini);

kharazi@sut.ac.ir (M. Kharazi); herfan@tabrizu.ac.ir (H.

Erfan-Niya)
}

doi: $10.24200 /$ sci.2019.50882.1918 or nanoplatelet, e.g., of clay or graphene [1-4]. However, due to the complex structure of the nanocomposites, identification of their structure and properties as well as design and optimization of their properties is still a major challenge for researchers. Growing need for new materials reveals the importance of new nanofillers and therefore, investigation into the effect of such nano-fillers on the structural, process, and physical properties comes to the first priority [5-8]. The physical properties, e.g., thermodynamic and mechanical, are strongly influenced by the structure and different phenomena in terms of dimensional and temporal aspects. In fact, spatial structure and shape of the polymer 
chain (configuration and conformation), mobility and dynamics of the chain, and many other phenomena have particular effects on the final properties of the compound [9-11].

On the other hand, incorporation of nanoscale fillers in the polymer increases the complexity of the system as well as the related properties. Considering the lack of experimental tools to provide detailed pictures of such structural aspects and dynamics of the chain and nano-fillers, using computational methods seems to be an answer to such deficiency. Traditionally, these methods work either on macroscale to predict the properties of materials or molecular scale to provide information about the scales down to the molecular dimensions. However, as described above, molecularscale characteristics affect the macroscale properties of the materials, especially when the fillers are nanoscalematerials. Here is exactly where the multi-scale computational methods have to be developed in which properties from down scales up to higher macroscale concepts and methods are covered step by step in order to transfer the properties from each scale to the upper one $[5,9,12]$.

At the molecular and atomic scales, computational methods have been developed, including molecular dynamics, molecular mechanics, and Monte Carlo methods of simulation $[5,10,12]$. At the higher scales, namely micro and meso scales, methods such as coarse grained molecular dynamics, Brownian dynamics, Lattice Boltzmann, and Langevin are commonly used for simulation [13-18]. Micro mechanical models such as Mori-Tanaka, Halipin-Tsai, and finite element and finite difference computational methods are also used in macro-scale simulations $[18,19]$. For the purpose of a multi-scale simulation, an algorithm to move topbottom or bottom-up is needed to describe how the information should be transferred between different scales.

In the present work, a nanocomposite system containing an epoxy resin and graphene as the nanofiller has been chosen. The resin is indeed a specific commercial epoxy-Novolac resin, namely SU8, with a wide range of applications as photoresist material in lithography process. SU8 is a high-temperature, chemically stable material with low toxicity and good transparency to visible light and is moderately lowcost [20-28]. Graphene was considered as the nanoscle single-sheet filler for the SU8 and investigated for its effect on the final properties of the SU8/graphene nanocomposites.

In the literature, several studies have been carried out on the simulation of polymers, nano-fillers, and polymer nanocomposites, including molecular dynamic simulation of epoxy resin $[29,30]$, simulation of graphene structure [31], investigation into the effect of graphene on polymeric nanocomposites [32-
38], and indeed, multi-scale simulation of polymers and polymeric nanocomposites [32,39-43]. Few works on the prediction of the properties of SU8 with the use of molecular dynamics simulation have also been published in the literature $[44,45]$.

However, there is not any report on the multi-scale simulation of SU8 and its nanocomposites and this is the subject of the present work, which is carried out based on the method developed by Rahman et al. [41]. During the simulation, many parameters of the system and the methodology should be optimized for use in our system, as will be shown in the subsequent sections.

\section{Multi-scale simulation of SU8 and SU8-graphene nanocomposite}

Based on the method proposed by Rahman et al. [41], the studied systems were simulated in three macro, meso, and molecular scales. Then, different scales of the system under study were linked through topbottom and bottom-up approaches so that the accuracy of results was investigated through data transferring between the subsequent scales.

The macro scale itself consisted of several scales from P0 (larger) to P3 (smaller) as depicted in Scheme 1. All the calculations and governing equations at these scales were drawn from the Peridynamics theory. At the lower scales, there was a shift toward lower scales, i.e., Coarse-Grained (CG) and molecular dynamics (A), as shown in Scheme 1. Therefore, there was a stepwise scale reduction as $\mathrm{P} 0>\mathrm{P} 1>\mathrm{P} 2>$ $\mathrm{P} 3>\mathrm{CG}>\mathrm{A}$ with the need for data transferring between the adjacent scales so that the properties from each scale could be read in the next scale as the input to the calculations carried out in the latter scale. Data

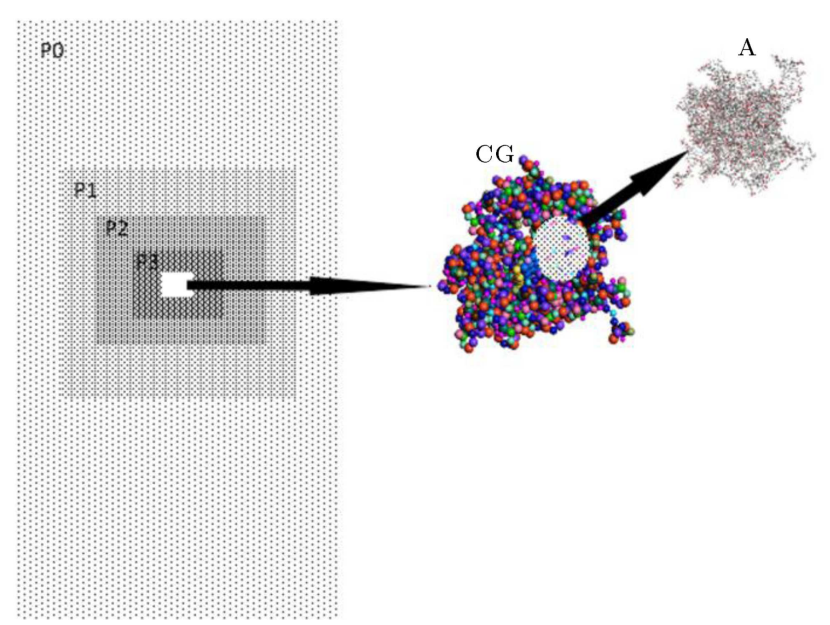

Scheme 1. Dividing material into different length scales for multi-scale simulations with P0 to P3 in macro scale, CG (coarse grained), and A (molecular scale). 
transferring could also be done by either top-bottom or bottom-up methodology.

\subsection{Top-bottom method}

In top-bottom method at $\mathrm{P} 0$ level, a two-dimensional plane with dimensions of $\mathrm{P} 0=10000 \AA \times 5000 \AA$, which is fixed in the lower part, is considered. According to the peridynamic theory, the $\mathrm{P} 0$ plane is set to accommodate 7875 number of particles, namely peridynamic particles interacting within a cutoff distance of $400 \AA$. To the free end of the plane, a velocity field of $0.1 \AA / \mathrm{fs}$ is applied in $Y$-axis direction for 100 steps. The new position of the particles is then determined by applying the NVE ensemble. Based on the plane deformation, the developed internal pressure in the system can be calculated using virial relationship by Eq. (1):

$$
\langle P\rangle=\frac{1}{2 A}\left(\sum_{i=1}^{m} r_{i} \bullet f_{i}\right),
$$

where $m$ is the number of peridynamic particles, $A$ is the area of the two-dimensional plane, $r_{i}$ is the location vector of each particle, and $f_{i}$ is the applied force to each particle caused by the interactions in the system. The calculated internal pressure in P0 is now applied as the external pressure to the lower peridynamic scale, $\mathrm{P} 1=500 \AA \times 500 \AA \times 500 \AA$, as shown in Scheme 2 . Then, $\mathrm{P} 1$ is brought to the equilibrated state in terms of energy by NVE ensemble, which is followed by another NPT ensemble with the applied external pressure. All these steps result in deformation of $\mathrm{P} 1$, leading to a newly developed internal pressure, which can be calculated using the virial relationship according to Eq. (2):

$$
\langle P\rangle=\frac{1}{3 V}\left(\sum_{i=1}^{m} r_{i} \bullet f_{i}\right),
$$

where $V$ is the volume of the simulation cell and other parameters are as in Eq. (1).

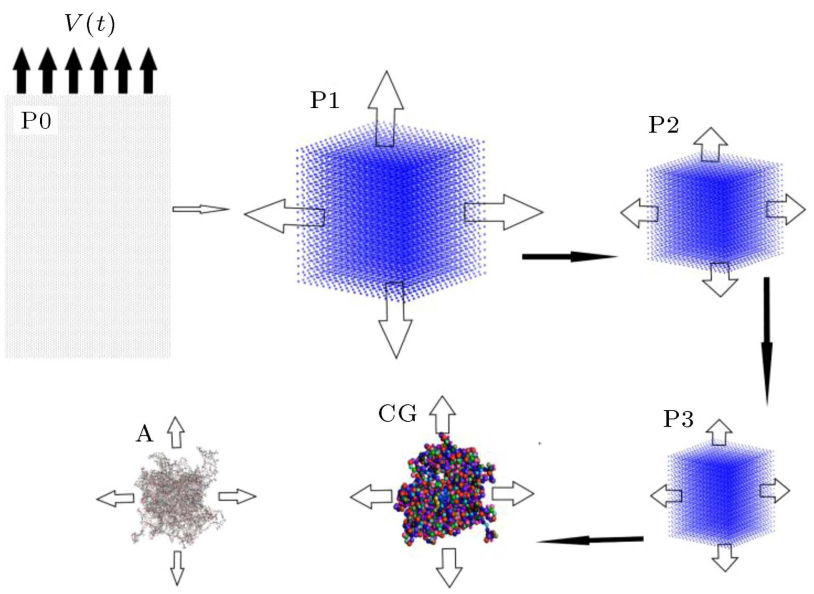

Scheme 2. Top-bottom method for different scales.
The calculated pressure in $\mathrm{P} 1$ is now applied as the external pressure to the next lower scale, P2 $=300 \AA \times 300 \AA \times 300 \AA$, and its internal pressure variation is calculated. This methods is followed to move from upper scale to the lower one, i.e., the internal pressure created by the deformation under external pressure is applied as the external pressure to the lower scales down to molecular scale A, as shown in Scheme 2. Dimensions of the other scales are as follows: $\mathrm{P} 3=200 \AA \times 200 \AA \times 200 \AA, \mathrm{CG}=50 \AA \times 50 \AA \times 50 \AA$ and $\mathrm{A}=45 \AA \times 45 \AA \times 45 \AA$.

All simulations in micro scales were performed by PDLAMMPS software [46,47] and LAMMPS was used for the meso-molecular scales [48].

\subsection{Bottom-up method}

In the bottom-up approach, the simulation box at the higher scale is investigated by the induced changes from the lower scale, e.g., the molecular scale. Obviously, in this case, for multi-scale data transferring from lower to the higher scales, defining an intermediate system is essential. In order to do this, each of the simulation cells in A, CG, and P3 levels is divided into 27 simulation sub-cells, as shown in Scheme 3. Then, the physical center of each sub-cell is calculated and a virtual particle (ghost atom) with the mass equivalent to the sub-cell is placed in the center. The physical center of each sub-cell is obtained according to Eq. (3):

$$
x_{j k}=\frac{\sum_{i=1}^{N_{j}} x_{i k}}{N_{j}},
$$

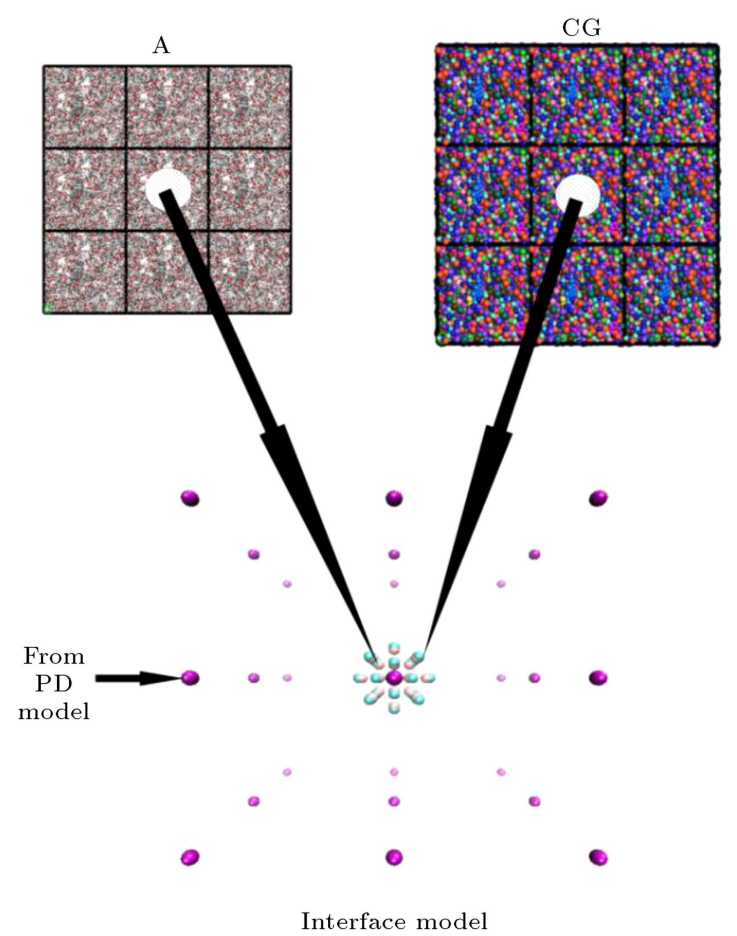

Scheme 3. Schematic representation of the formation of intermediate system in the bottom-up method. 
where $x_{j k}$ represents the virtual particle coordinates in the cell $j$ and $N_{j}$ is the number of atoms in the cell $j$.

In the next step, the virtual particles associated with each of the scales, without changing their coordinates, are assembled into an intermediate model, as shown in Scheme 3. The interactions between the virtual particles of model $\mathrm{A}$ and the $\mathrm{CG}$ model are calculated using the Lennard-Jones model. The average velocity of the virtual particles of peridynamic model in the previous step is also calculated and applied as the boundary conditions to the virtual particles of the CG model. For the sake of brevity, the readers are referred to [35] for the equations used in several calculations.

The virtual particles of the Coarse Grained system (CG) are exposed to NVE and NPT ensembles so that they reach an equilibrium state and the dimensions of the simulation box are changed. In this case, the force is introduced from the virtual particles of $\mathrm{CG}$ into peridynamic particles $\mathrm{P} 3$, which also changes the position of the peridynamic particles under the NVE and NPT ensembles. The overall deformation gradient based on the cell dimensional variations is applied to the core of the upper scale P2. Finally, the system undergoes equilibrium state by NVE and NPT runs, during which the simulation box reaches new dimensions.

Similarly, in this case, the gradient of deformation is calculated and applied to a simulated system at the higher scale. Thus, the deformation generated at P2 is transferred to $\mathrm{P} 1$ and then, from $\mathrm{P} 1$ to $\mathrm{P} 0$. Whenever the deformations created in the bottom-up movement at any given scale are in the range of what was obtained in the top-bottom approach, the validity of the multiscale simulation is confirmed, which indeed proves the success of the methodology employed for the multiscale simulation.

\section{Simulation of SU8}

Before proceeding to multi-scale simulation of SU8/ graphene nanocomposites, the SU8 itself has to be simulated by a multi-scale simulation procedure. This is necessary because SU8 is a multifunctional molecule which undergoes crosslinking by UV irradiation with the appropriate crosslinking agent and photoinitiator. This will be described in the following sections.

\subsection{Simulation of $S U 8$ in molecular scale}

First, a representative molecular structure has to be determined to resemble the crosslinked SU8. According to the results of Tam and Lau [44,45], Zhang et al. [49], and Mohammadzadeh Honarvar et al. [50], a number of molecular structures for the crosslinked SU8 have been proven to exist, which are the building blocks for our purpose. Therefore, different structures were proposed, which are shown in Figure 1. All the proposed
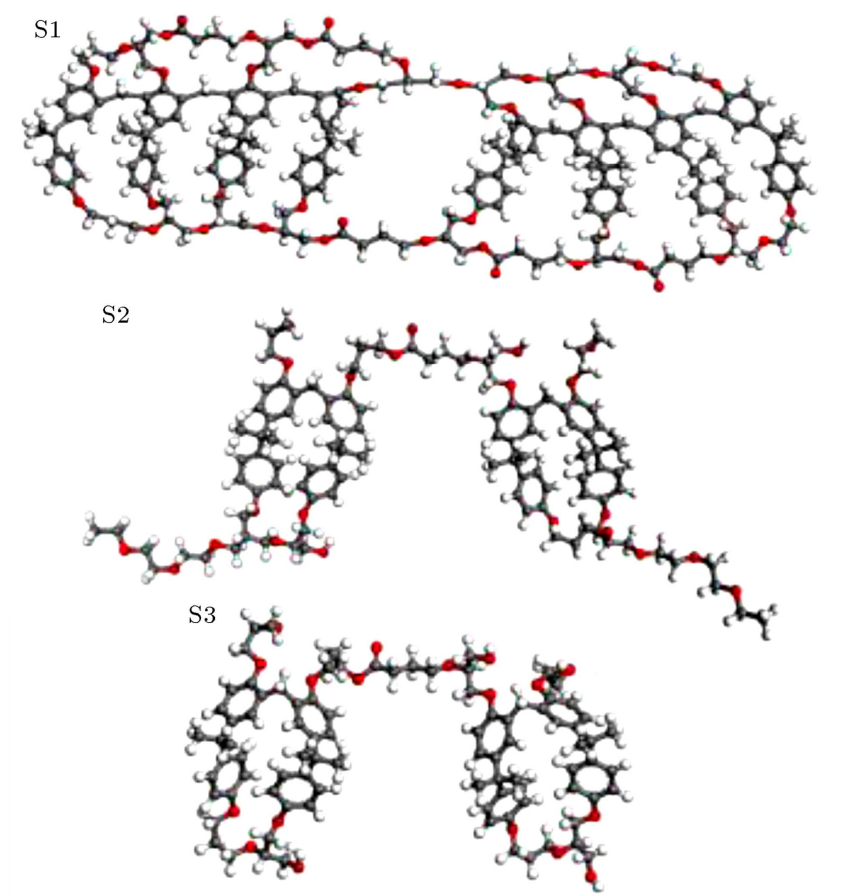

Figure 1. Chemical structures prepared to simulate SU8. In all cases, carbon, hydrogen, and oxygen atoms are marked with gray, white, and red colors, respectively.

structures were subject to the energy minimization process to reach the lowest level of energy and to obtain the optimized molecular structure. At the final stage, the S3 structure (Figure 1) with the lowest energy level amongst all the structures was selected to represent the SU8 structure. Therefore, the structure is denoted by SU8 through the text. A three-dimensional simulation cell was built up consisting of 40 SU8 molecules (8428 atoms) with alternating (periodic) boundary conditions in all directions (Figure 2). The interactions in the system were calculated using the pcff force field with a cutoff distance of $8 \AA$.

Initially, in order to optimize the SU8, the simulation cell was subjected to 100000 steps of energy minimization followed by MD runs under isobaric isothermal ensemble (NPT) at $298^{\circ} \mathrm{K}$. Without applying any external pressure, the system reached its equilibrated state of energy, temperature, and density. The final density of the system was calculated to be $0.91 \mathrm{~g} / \mathrm{cm}^{3}$, which was in the range of the reported values for density [44,50-52]. Finally, in order to ensure the stability of the system, NVT ensemble was utilized, which is evident in the temperature and energy profiles shown in Figures 3 and 4 . Velocity-Verlet integrator was used to integrate the differential equations of motion throughout the simulation steps.

\subsection{Simulation of SU8 at meso scale}

At the meso scale, the coarse-grained method was used to simulate the chemical structure so that SU8 


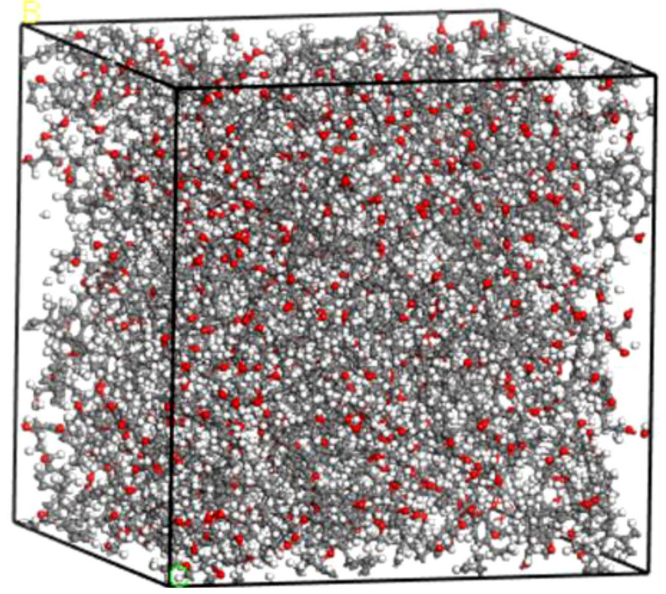

Figure 2. Snapshot of the simulation cell of SU8. Carbon, hydrogen, and oxygen atoms are marked with gray, white, and red colors, respectively.

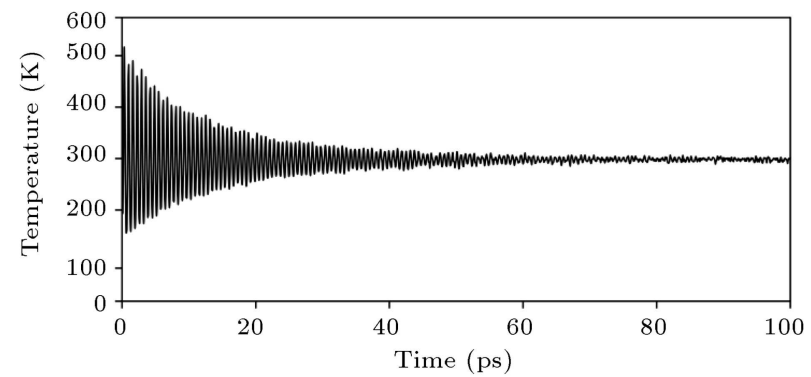

(a)

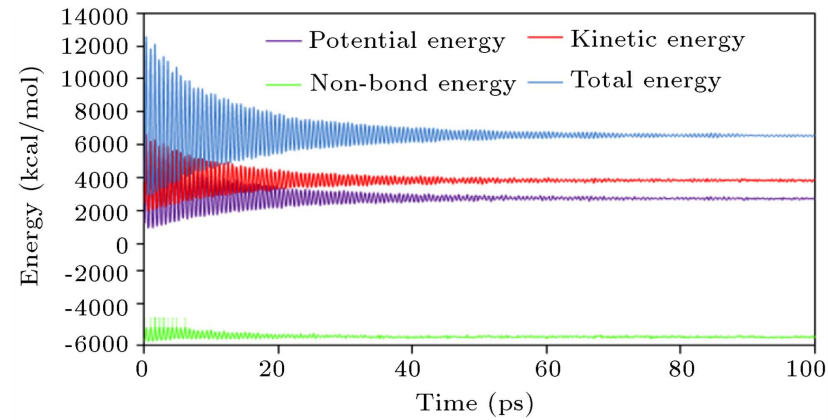

(b)

Figure 3. Temperature profile (a) and energy (b) of NPT at molecular scale.

structure was rendered into a bead-spring model by replacing some of the atoms with an equivalent ball, as demonstrated in Scheme 4. The balls then represented the beads in the coarse-grained modeling of the materials.

Therefore, in the simulation cell, the beads were composed instead of molecular parts as shown in Figure 5. The figure shows a cell containing 56 SU8 molecules with periodic boundary conditions. At this stage, the interactions between the beads were of Lenard Jones type. The cell was brought to the optimized and equilibrated state by energy minimization through sequential NPT and NVE ensembles, as the

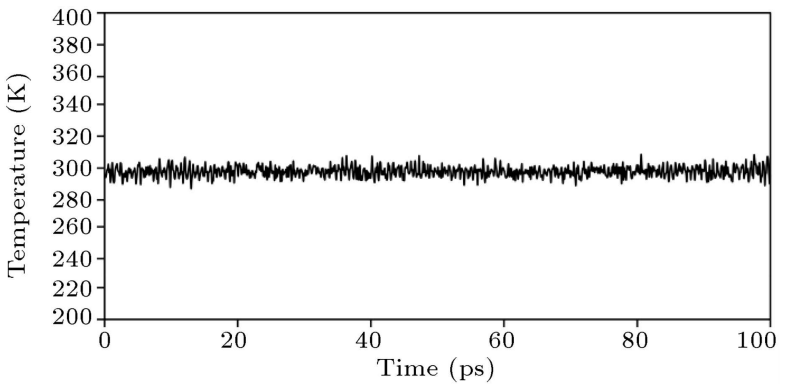

Figure 4. Temperature profile of NVT run at molecular scale.

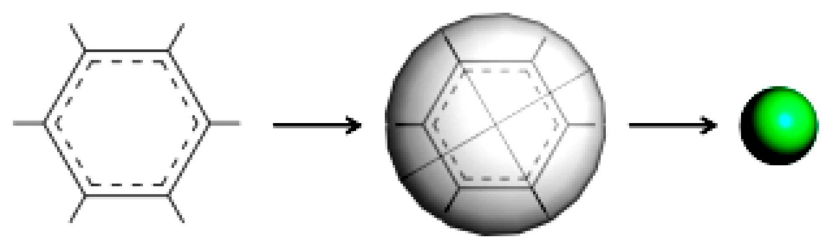

Scheme 4. A sample of the process of converting an atomic structure of a molecular model to an equivalent bead of a coarse-grained system
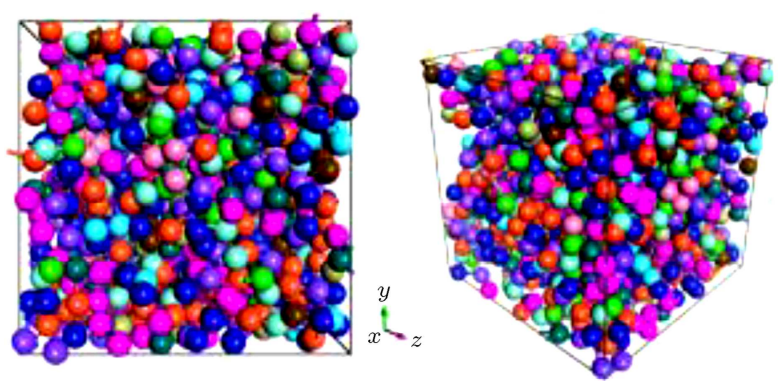

Figure 5. SU8 simulation cell of coarse grained system at meso scale.

temperature and energy profiles in Figures 6 and 7 show.

The meso scale simulation was indeed the bridging step, which transferred the data from molecular scale to the macro scale.

\subsection{SU8 simulation in macro scale}

At macro scale, SU8 was simulated in 4 different dimensions (namely $\mathrm{P} 0, \mathrm{P} 1, \mathrm{P} 2$, and $\mathrm{P} 3$ ) according to the peridynamic theory with the operational details described in Section 2. Since the LPS (linear peridynamic solid) model had been selected, numerical values of bulk modulus, shear modulus, and crosslinked SU8 density were selected from references, which were equal to $3.14 \mathrm{GPa}, 1.2 \mathrm{GPa}$, and $1.2 \mathrm{~g} / \mathrm{cm}^{3}$, respectively $[21,52]$.

At $\mathrm{P} 0$ level, a total number of 7875 peridynamic particles with $400 \AA$ cutoff distance; at P1 level, 4913 particles with a cutoff distance of $90 \AA$; at P2 level, a total number of 3375 particles with a cutoff distance of $54 \AA$; and finally, at P3 level, 5832 peridynamic 


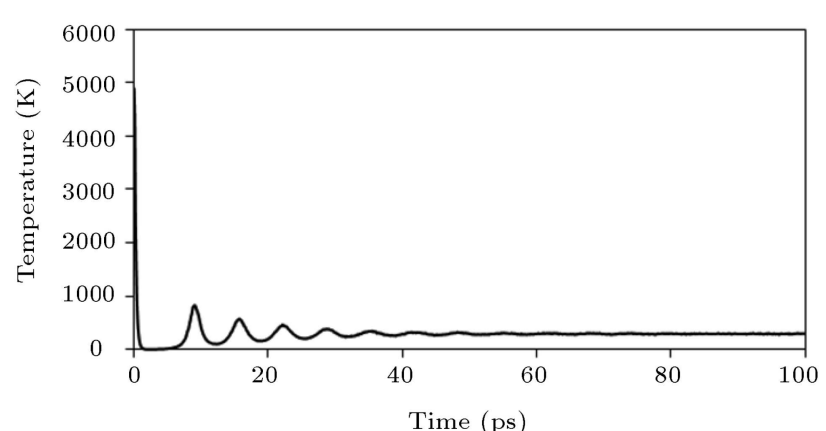

(a)

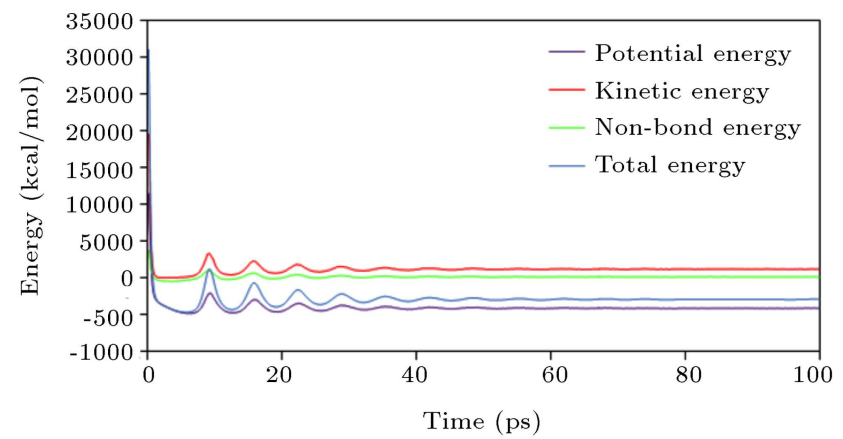

(b)

Figure 6. Temperature profile (a) and energy (b) of meso-scale NPT for SU8.

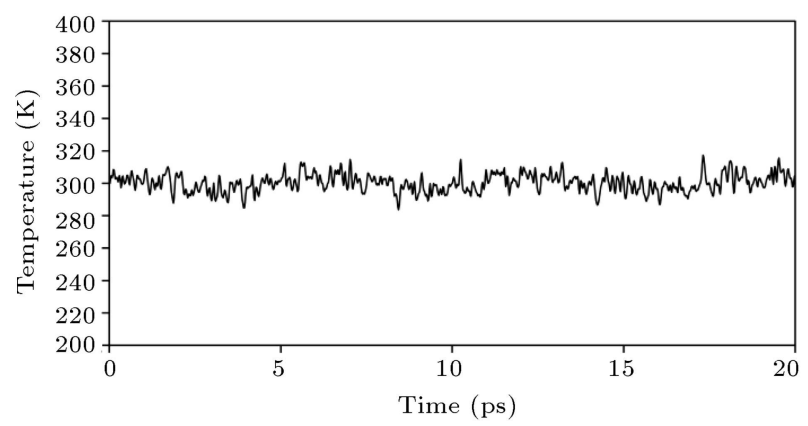

Figure 7. Temperature profile of NVT at meso scale for SU8.

particles with a cutoff distance of $40 \AA$ were selected. All the 4 levels of simulation with the peridynamic particles of each level constructed a three-dimensional cube as shown in Figure 8. At each level, the structure was subjected to energy minimization in order to optimize and stabilize the system.

\subsection{Results of multi-scale simulation of $S U 8$}

According to the methodology described in Section 2, in the top-bottom approach, a velocity field has to be applied in $Y$ direction to the free edge of the P0 plane. This led to $0.01 \%$ deformation of the plane with internal pressure development, which was calculated to be $20.6 \mathrm{~atm}$, in this study. This was indeed the external pressure applied to $\mathrm{P} 1$ in the next step, leading to a deformation of $0.772 \%$ and an internal pressure of $15.4 \mathrm{~atm}$. For the next levels, namely P2, P3, CG, and

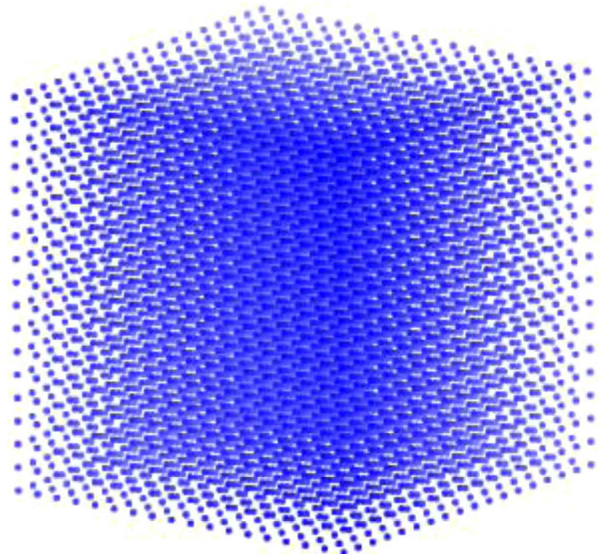

Figure 8. Snapshot of SU8 simulation cell using a peridynamic technique.

A, the deformation values and internal pressures were $0.015 \%$ and $13.3 \mathrm{~atm}, 0.053 \%$ and $79.4 \mathrm{~atm}, 0.28 \%$ and $32 \mathrm{~atm}$, and $1.3 \%$ and 30.9 atm, respectively.

In the bottom-up approach, the displacement gradient was obtained and applied to the $\mathrm{P} 2$ core before NPT and NVE stabilizing runs. The system was at equilibrium state. The dimensions of the simulation cell were deformed to ca $0.0105 \%$ in P2. Subsequently, the displacement gradient from $\mathrm{P} 2$ was applied to the core of $\mathrm{P} 1$, leading to a deformation of ca $0.077 \%$. This was applied, in the next step, to the core of $\mathrm{P} 0$, leading to a displacement gradient of ca $0.0107 \%$. Comparison of the values of deformation gradients in the corresponding levels of both top-bottom and bottom-up approaches confirms the validity of the simulation algorithm and the parameter selection.

\section{Simulation of SU8-graphene nanocomposite}

In this section, in order to simulate SU8-graphene nanocomposite, the graphene structure is first simulated, before multi-scale simulation of SU8-graphene nanocomposite. As discussed earlier, the macro scale simulation of SU8, shear modulus, and density are the necessary parameters that have to be determined. For this purpose, the results of the simulation of SU8/graphene nanocomposites by molecular dynamics methods were used. However, the accuracy of the used data was evaluated at the end of the multiscale simulation of the nanocomposite (the data from completed simulation).

\subsection{Simulation of SU8-graphene nanocomposite at molecular scale}

The molecular structure of a single-layer graphene used in molecular dynamics simulation is shown in Figure 9. Its dimensions are $26.18 \times 13.337 \AA$. Using this graphene and SU8, a simulation cell containing 2.1 wt.\% graphene was constructed having periodic boundary conditions as shown in Figure 10. The 


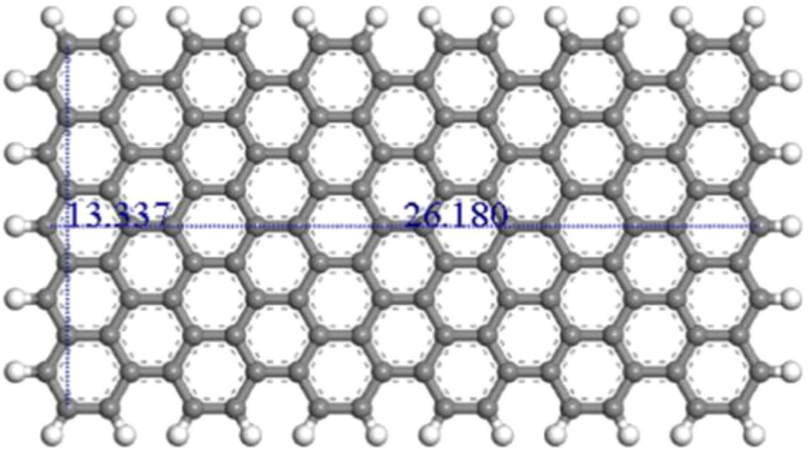

Figure 9. The simulated graphene. Carbon and hydrogen atoms are gray and white, respectively.

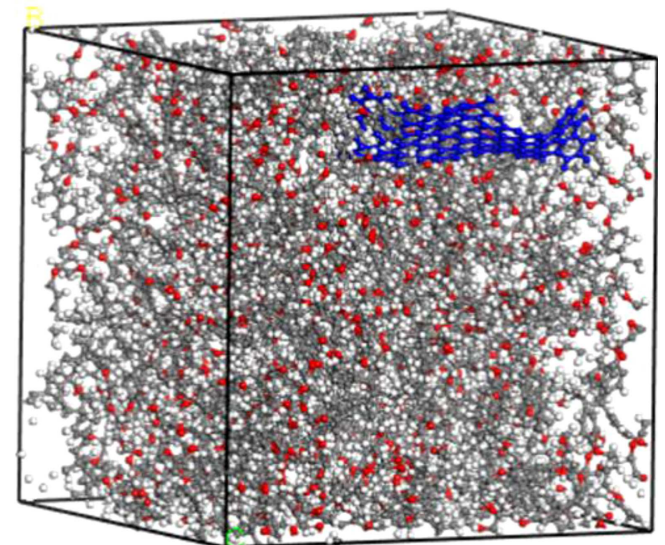

Figure 10. Snapshot of the SU8-graphene nanocomposite simulation cell; carbon, hydrogen, and oxygen atoms are blue, white, and red, respectively. The single-layer graphene can be seen on the top right corner of the box.

interactions in the system were calculated using the pcff force field at $8 \AA$ cutoff distance. Similar to Section 3.1, the nanocomposite underwent energy minimization under NPT conditions. The final density of the nanocomposite was calculated at $1.1 \mathrm{~g} / \mathrm{cm}^{3}$.

The effect of graphene on the mechanical properties of SU8 was calculated using the method developed by Theodorou and Suter [53].

The following steps were taken to calculate the mechanical properties:

1. Optimizing and stabilizing the desired structure by energy minimization;

2. Applying strain (less than 1\%) to the system;

3. Minimizing the energy of the deformed structure;

4. Repeating steps 1 to 3 for the next optimized configuration by the MD runs;

5. Calculating the mechanical properties by average all over the simulation cells for the structure under consideration.

The elastic constants were calculated using the second derivative of the internal energy $(U)$ with respect to the applied deformation according to Eq. (4):

$$
C_{i j}=\frac{1}{V} \frac{\partial^{2} U}{\partial \varepsilon_{i} \partial \varepsilon_{j}}=\frac{\partial \sigma_{i}}{\partial \varepsilon_{j}} .
$$

The Lame constants were calculated using Eqs. (5) and (6):

$$
\begin{aligned}
& \lambda=\frac{1}{3}\left(C_{11}+C_{22}+C_{33}\right)-\frac{2}{3}\left(C_{44}+C_{55}+C_{66}\right), \\
& \mu=\frac{1}{3}\left(C_{44}+C_{55}+C_{66}\right) .
\end{aligned}
$$

And the mechanical properties were obtained using Eqs. (7), (8), and (9):

$$
\begin{aligned}
& G=\mu, \\
& B=\lambda+\frac{2}{3} \mu, \\
& E=\mu \frac{3 \lambda+2 \mu}{\lambda+\mu},
\end{aligned}
$$

where $E$ is the Young's modulus, $B$ is the bulk modulus, $G$ is the shear modulus, $\lambda$ and $\mu$ are the Lamé constants.

The mechanical properties calculated for SU8 and SU8/graphene nanocomposites are summarized in Table 1 and compared graphically in Figure 11.

From the data in Table 1 and Figure 11, it is concluded that incorporation of graphene in SU8 results in $63 \%$ increase in the Young's modulus, $100 \%$ increase in the bulk modulus, and $80 \%$ increase in the shear modulus. These are the data used in multiscale simulation of SU8-graphene nanocomposite using the peridynamic theory, as discussed in the following sections.

\subsection{Simulation of SU8-graphene nanocomposite in meso scale}

Coarse grained model of SU8-graphene was constructed under the procedure explained in Section 3.2, as shown in Figure 12. In the simulation cell, there were 56 molecules of SU8 being packed in a $50 \AA \times 50 \AA \times 50$

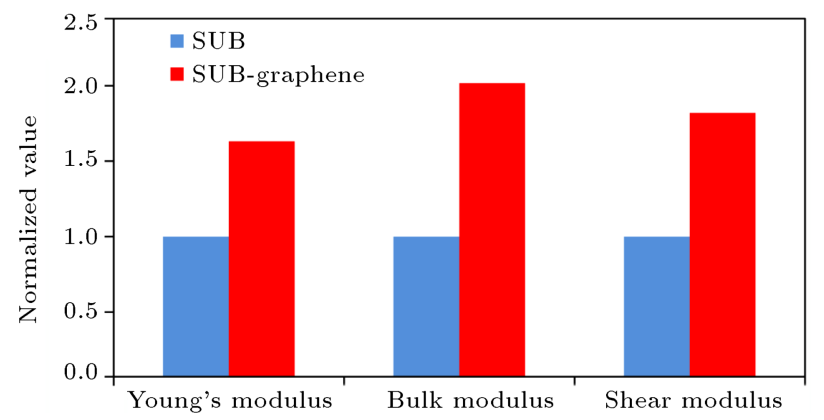

Figure 11. Mechanical properties $E, B$, and $G$ predicted for SU8 and its nanocomposite with graphene. 
Table 1. Mechanical properties predicted or calculated for SU8 and its nanocomposite with graphene.

\begin{tabular}{cccc}
\hline Sample & $\begin{array}{c}\text { Normalized } \\
\text { Young's modulus }\end{array}$ & $\begin{array}{c}\text { Normalized } \\
\text { bulk modulus }\end{array}$ & $\begin{array}{c}\text { Normalized } \\
\text { shear modulus }\end{array}$ \\
\hline SU8 & $1 \pm 0.039$ & $1 \pm 0.010$ & $1 \pm 0.013$ \\
SU8/graphene & $1.631 \pm 0.075$ & $2.017 \pm 0.012$ & $1.82 \pm 0.020$ \\
\hline
\end{tabular}
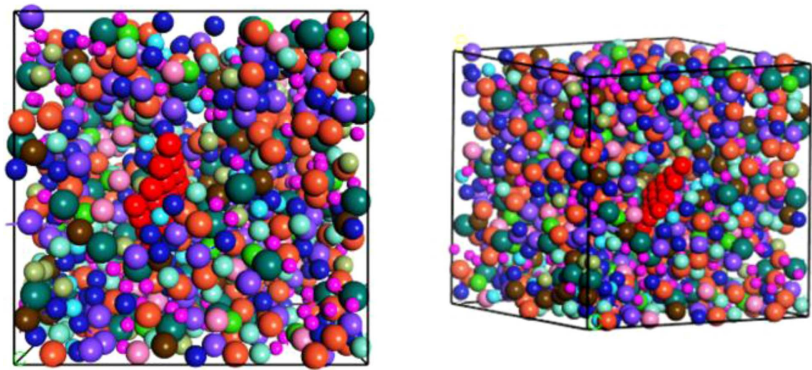

Figure 12. The simulation cell of SU8/graphene nanocomposite in the coarse grained model at meso scale. The graphene layer is marked with red.

$\AA$ cubic cell with periodic boundary condition in all directions. The governing interactions were of LenardJones potential field type. The cell underwent energy minimization to remove any overlapping and excess energy of the system until an optimized and stable state was reached. This was performed by 100000 steps of MD optimization under NPT conditions with no external pressure in order to equilibrate and adjust density.

\subsection{Simulation of SU8/graphene nanocomposite in macro scale}

As described in Section 3.3, the SU8/graphene nanocomposite was simulated using the peridynamic theory in 4 different scales (P0, P1, P2, and P3). Considering the LPS model, the required numerical values for bulk modulus, shear modulus, and density were obtained from molecular dynamics simulation (Section 4.1). The used values were 6.31, 2.18 GPa, and $1.21 \mathrm{~g} / \mathrm{cm}^{3}$, respectively.

\subsection{Results of multi-scale simulation of SU8/graphene nanocomposite}

In the top-bottom scaling method for the SU8/ graphene nanocomposite, the deformation developed in P0, P1, and P2 was $0.01 \%, 0.106 \%$, and $0.018 \%$, respectively. These values, in performing the bottomup scaling method, were $0.0165 \%$ for $\mathrm{P} 2,0.113 \%$ for $\mathrm{P} 1$, and $0.0085 \%$ for $\mathrm{P} 0$. Therefore, due to the similarity of the values of deformation in both scaling methods of top-bottom and bottom-up, the simulation methodology is confirmed.

\section{Conclusion}

In this study, a multi-scale simulation of SU8 and its nanocomposite comprising 2.1 wt.\% graphene was performed through linking simulations in different scales of atomistic, meso, and macro. MD, coarse grain $\mathrm{MD}$, and $\mathrm{PD}$ coupled with MD (PDLAMMPS) were used to simulate the system in atomistic, meso, and macro scales, respectively. LJ interaction was used to define intermolecular interactions for atomistic and coarse grain systems. Interactions in macro scale simulation were defined based on peridynamic formulations. For this purpose, first, suitable molecular structures for SU8 and graphene were designed in molecular scales. By the selection of LPS model in the peridynamic method, the numerical values of the bulk modulus, shear modulus, and density for SU8 were determined based on the experimental results reported in the literature. However, in the case of SU8/graphene nanocomposite, a method was developed to estimate the physical-mechanical properties based on the method of Theodorou et al. in atomistic scale [51]. The multi-scale simulation was carried out in both top-bottom and bottom-up types and this helped to validate the techniques as the extents of deformation were found to be almost equal independent of scaling down or up.

In order to extend the method to any desired material, determining the parameters of the material and the corresponding chemical structures in the new system are required. However, the basic theory of the methodology remains the same.

\section{Acknowledgment}

The authors wish to express their gratefulness to Iran Nanotechnology Initiative Council for their partial financial support of the work under contract no. of 71734. The authors, would also like to gratitude Sahand University of Technology which, the present work could not be conducted without supports receiving from them.

\section{References}

1. Okada, A. and Usuki, A. "Twenty years of polymer-clay nanocomposites", Macromol. Mater. Eng., 291(12), pp. 1449-1476 (2006).

2. Ray, S.S. and Okamoto, M. "Polymer/layered silicate nanocomposites: a review from preparation to processing", Prog. Polym. Sci., 28(11), pp. 1539-1641 (2003).

3. Tjong, S.C. "Structural and mechanical properties of 
polymer nanocomposites", Mater. Sci. Eng. R Rep., 53(3), pp. 73-197 (2006).

4. Mehdikhani-Nahrkhalaji, M., Tavakoli, E., and Zargar-Kharazi, A. et al. "A novel nano-composite scaffold for cartilage tissue engineering", Sci. Iran., 25(3), pp. 1815-1823 (2018).

5. Costanzo, F. and Gray, L.G. "A micromechanicsbased notion of stress for use in the determination of continuum-level mechanical properties via molecular dynamics", In Multiscale Modeling and Simulation of Composite Materials and Structures, Y.W. Kwon, D.H. Allen, and R. Talreja, Eds., 1st Edn, pp. 203-235, Springer, New York, USA (2008).

6. Zeng, Q., Yu, A., and Lu, G. "Multiscale modeling and simulation of polymer nanocomposites", Prog. Polym. Sci., 33(2), pp. 191-269 (2008).

7. Zeng, Q., Yu, A., Lu, G., et al. "Clay-based polymer nanocomposites: research and commercial development", J. Nanosci. Nanotechnol., 5(10), pp. 1574-1592 (2005).

8. Zeng, Q., Yu, A., and Lu, G.M. "Interfacial interactions and structure of polyurethane intercalated nanocomposite", Nanotechnology, 16(12), pp. 27572763 (2005).

9. Gates, T., Odegard, G., and Frankland, S. et al. "Computational materials: multi-scale modeling and simulation of nanostructured materials", Compos. Sci. Technol., 65(15), pp. 2416-2434 (2005).

10. Smith, J., Bedrov, D., Borodin, O., et al. "Multiscale modeling of polymer based nanomaterials", NSTI Nanotech., Boston, USA, pp. 724-727 (2006).

11. Yan, L.-T., and Xie, X.-M. "Computational modeling and simulation of nanoparticle self-assembly in polymeric systems: Structures, properties and external field effects", Prog. Polym. Sci., 38(2), pp. 369-405 (2013).

12. Efendiev, Y. and Hou, T.Y. "Applications of multiscale finite element methods", In Multiscale Finite Element Methods: Theory and Applications, S.S. Antman, J.E. Marsden, and L. Sirovich, Eds., 1st Edn, pp. 95-163, Springer, New York, USA (2009).

13. Smith, G.D., Bedrov, D., Li, L., et al. "A molecular dynamics simulation study of the viscoelastic properties of polymer nanocomposites", J. Chem. Phys., 117(20), pp. 9478-9489 (2002).

14. Carter, E.A. "Challenges in modeling materials properties without experimental input", Science, 321(5890), pp. 800-803 (2008).

15. Elliott, J. "Novel approaches to multiscale modelling in materials science", Int. Mater. Rev., 56(4), pp. 207225 (2011).

16. Harmandaris, V.A., Floudas, G., and Kremer, K. "Temperature and pressure dependence of polystyrene dynamics through molecular dynamics simulations and experiments", Macromolecules, 44(2), pp. 393-402 (2010).
17. Schneider, G., Nusser, K., Willner, L., et al. "Dynamics of entangled chains in polymer nanocomposites", Macromolecules, 44(15), pp. 5857-5860 (2011).

18. Valavala, P. and Odegard. G. "Modeling techniques for determination of mechanical properties of polymer nanocomposites", Rev. Adv. Mater. Sci., 9, pp. 34-44 (2005).

19. Baghani, M., Dolatabadi, R., and Baniassadi, M. "Developing a finite element beam theory for nanocomposite shape-memory polymers with application to sustained release of drugs", Sci. Iran., Trans. B., 24(1), pp. 249-259 (2017).

20. Hamlett, C.A.E., McHale, G., and Newton, M.I. "Lithographically fabricated SU8 composite structures for wettability control", Surf. Coat. Technol., 240, pp. 179-183 (2014).

21. Blagoi, G., Keller, S., Persson F., et al. "Photochemical modification and patterning of SU-8 using anthraquinone photolinkers", Langmuir, 24(18), pp. 9929-9932 (2008).

22. Rodríguez-Ruiz, I., Llobera, A., Vila-Planas, J., et al. "Analysis of the structural integrity of SU-8-based optofluidic systems for small-molecule crystallization studies", Anal. Chem., 85(20), pp. 9678-9685 (2013).

23. Hu, M., Guo, Q., Zhang, T., et al. "SU-8-induced strong bonding of polymer ligands to flexible substrates via in situ cross-linked reaction for improved surface metallization and fast fabrication of highquality flexible circuits", ACS Appl. Mater. Inter., 8(7), pp. 4280-4286 (2016).

24. Romeo, A., Liu, Q., Suo, Z., et al. "Elastomeric substrates with embedded stiff platforms for stretchable electronics", Appl. Phys. Lett., 102(13), p. 131904 (2013).

25. Rahiminejad, S., Pucci, E., Haasl, S., et al. "SU8 ridgegap waveguide resonator", Int. J. Microw. Wirel. T., 6(05), pp. 459-465 (2014).

26. Nagaiyanallur, V.V., Kumar, D., Rossi, A., et al. "Tailoring SU-8 surfaces: covalent attachment of polymers by means of nitrene insertion", Langmuir, 30(3), pp. 10107-10111 (2014).

27. Tian, Y., Shang, X., Wang, Y., et al. "Investigation of SU8 as a structural material for fabricating passive millimeter-wave and terahertz components", J. Micro/Nanolithogr. MEMS. MOEMS., 14(4), pp. 044507044509 (2015).

28. Mehboudi A. and Yeom, J. "A two-step sealing-andreinforcement SU8 bonding paradigm for the fabrication of shallow microchannels", J. Micromech. Microeng., 28(3), p. 035002 (2018).

29. $\mathrm{Wu}, \mathrm{C}$, and $\mathrm{Xu}, \mathrm{W}$. "Atomistic molecular simulations of structure and dynamics of crosslinked epoxy resin", Polymer, 48(19), pp. 5802-5812 (2007).

30. Wu, C. and Xu, W. "Atomistic molecular modelling of crosslinked epoxy resin", Polymer, 47(16), pp. 60046009 (2006). 
31. Ansari, R., Ajori, S., and Motevalli, B. "Mechanical properties of defective single-layered graphene sheets via molecular dynamics simulation", Superlattices $M i$ crostruct., 51(2), pp. 274-289 (2012).

32. Ebrahimi, S., Ghafoori-Tabrizi, K., and Rafii-Tabar, H. "Multi-scale computational modelling of the mechanical behaviour of the chitosan biological polymer embedded with graphene and carbon nanotube", Comp. Mater. Sci., 53(1), pp. 347-353 (2012).

33. Rissanou, A.N. and Harmandaris, V. "Structure and dynamics of poly (methyl methacrylate)/graphene systems through atomistic molecular dynamics simulations", J. Nanopart. Res., 15(5), p. 1589 (2013).

34. Rissanou, A.N. and Harmandaris, V. "Dynamics of various polymer-graphene interfacial systems through atomistic molecular dynamics simulations", Soft Matter, 10(16), pp. 2876-2888 (2014).

35. Rahman, R. and Haque, A. "Molecular modeling of crosslinked graphene-epoxy nanocomposites for characterization of elastic constants and interfacial properties", Compos. Part B-Eng., 54, pp. 353-364 (2013).

36. Alian, A., Dewapriya, M., and Meguid, S. "Molecular dynamics study of the reinforcement effect of graphene in multilayered polymer nanocomposites", Mater. Design, 124, pp. 47-57 (2017).

37. Güryel, S., Walker, M., Geerlings, P., et al. "Molecular dynamics simulations of the structure and the morphology of graphene/polymer nanocomposites", Phys. Chem. Chem. Phys., 19(20), pp. 12959-12969 (2017).

38. Sun, R., Li, L., and Feng, C. et al. "Tensile behavior of polymer nanocomposite reinforced with graphene containing defects", Eur. Polym. J., 98, pp. 475-482 (2018).

39. Yu, S., Yang, S., and Cho, M. "Multi-scale modeling of cross-linked epoxy nanocomposites", Polymer, 50(3), pp. 945-952 (2009).

40. Yu, S., Yang, S., and Cho, M. "Multiscale modeling of cross-linked epoxy nanocomposites to characterize the effect of particle size on thermal conductivity", $J$. Appl. Phys., 110(12), p. 124302 (2011).

41. Rahman, R. and Haque, A. "A peridynamics formulation based hierarchical multiscale modeling approach between continuum scale and atomistic scale", Int. J. Comput. Mater. Sci. Eng., 1(03), p. 1250029 (2012).

42. Kim, B., Choi, J., Yang, S., et al. "Multiscale modeling of interphase in crosslinked epoxy nanocomposites", Composites, Part B, 120, pp. 128-142 (2017).

43. Lin, F., Yang, C., Zeng, Q., et al. "Morphological and mechanical properties of graphene-reinforced PMMA nanocomposites using a multiscale analysis", Comput. Mater. Sci., 150, pp. 107-120 (2018).

44. Tam, L.-h. and Lau, D. "A molecular dynamics investigation on the cross-linking and physical properties of epoxy-based materials", RSC Adv., 4(62), pp. 3307433081 (2014).
45. Tam, L.-H. and Lau, D. "Moisture effect on the mechanical and interfacial properties of epoxy-bonded material system: An atomistic and experimental investigation", Polymer, 57, pp. 132-142 (2015).

46. Parks, M.L., Seleson, P., Plimpton, S.J., et al. "Peridynamics with LAMMPS: A user guide, v0. 3 Beta", Sandia Report (2011-8253), pp. 1-34 (2011).

47. Parks, M.L., Lehoucq, R.B., Plimpton, S.J., et al. "Implementing peridynamics within a molecular dynamics code", Comput. Phys. Commun., 179(11), pp. 777-783 (2008).

48. Plimpton, S., Crozier, P., and Thompson, A. "LAMMPS-large-scale atomic/molecular massively parallel simulator", Sandia National Laboratories, 18, p. 43 (2007).

49. Zhang, J., Chan-Park, M.B., and Li, C.M. "Network properties and acid degradability of epoxy-based SU8 resists containing reactive gamma-butyrolactone", Sensor. Actuat. B-Chem., 131(2), pp. 609-620 (2008).

50. Mohammadzadeh Honarvar, F., Pourabbas, B., Salami Hosseini, M., et al. "Molecular dynamics simulation: The effect of graphene on the mechanical properties of epoxy based photoresist: SU8", Sci. Iran., 25(3). pp. 1879-1890 (2018).

51. Hossenlopp, J., Jiang, L., Cernosek, R., et al. "Characterization of epoxy resin (SU-8) film using thicknessshear mode (TSM) resonator under various conditions", J. Polym. Sci. Pol. Phys., 42(12), pp. 23732384 (2004).

52. Suter, M., Ergeneman, O., Zürcher, J., et al. "A photopatternable superparamagnetic nanocomposite: Material characterization and fabrication of microstructures", Sensor. Actuat. B-Chem., 156(1), pp. 433-443 (2011).

53. Theodorou, D.N. and Suter, U.W. "Atomistic modeling of mechanical properties of polymeric glasses", Macromolecules, 19(1), pp. 139-154 (1986).

\section{Biographies}

Faraz Mohammadzadeh Honarvar is a last-year $\mathrm{PhD}$ candidate in Polymer Engineering at Sahand University of Technology, Tabriz, Iran. He received BSc degree from Amirkabir University of Technology (Tehran Polytechnic), Tehran, Iran, and MSc degree from University of Tehran, Tehran, Iran, both in Polymer Engineering. His research fields are nanocomposites, mechanical properties of nanocomposites, and simulation of nanocomposites in molecular and macro scales. Also, he is interested in multi-scale simulation.

Behzad Pourabbas received his $\mathrm{PhD}$ in Polymer Science and Technology from University of Shiraz, Shiraz, Iran, in 1996. He joined the Department of Chemical Engineering at Sahand University of Technology in Tabriz, Iran, as an Assistant Professor 
in the same year and since then, he has been a member of the faculty. He was promoted to the Associate Professor position in 2005 and later on, to Full Professor position in 2009. In 2008, when the Department of Polymer Engineering started its activities independently, he joined the department and worked as its first head. During his academic career, Dr. Pourabbas has taught Physical Chemistry of Polymers, Polymer Physics, Polymer Characterization Methods, and Organic Chemistry. His main research activities focus on nanostructured materials, surface and surface modification, nano-microfabrication, developing polymers, and electronic materials.

Mahdi Salami Hosseini received his BSc and MSc degrees from Amirkabir University of Technology in 2001 and 2004, respectively. He received his PhD in Polymer Engineering from the same university in 2009. He joined Polymer Engineering Department of Sahand University of Tecnology as Assistant Professor in 2009 and became Associate Professor in 2015. His current research interests include process simulation and numerical rheology using Finite Element Method (FEM) and Stokesian Dynamics (SD) focusing on microfluidic devices.

Mahsa Kharazi received her $\mathrm{PhD}$ degree in
Aerospace Engineering from Amirkabir University of Technology (Tehran Polytechnic), Tehran, Iran, in 2008. In 2008, she joined the Department of Mechanical Engineering, Sahand University of Technology, Tabriz, Iran, as an Assistant Professor and since then, she has been a faculty member. She became an Associate Professor in 2017. Her teaching areas are Continuum Mechanics, Plasticity, Finite Element Method, and Stability of Structures.

Her $\mathrm{PhD}$ thesis was on the stability analysis of delaminated composite materials and over the last five years, she has been involved with damage modeling and analysis in solid mechanics. Her current research interests include structural stability, damage analysis, numerical methods, and non-local theories.

Hamid Erfan-Niya received the $\mathrm{PhD}$ degree in Chemical Engineering in 2011 from Amirkabir University of Technology (Tehran Polytechnic), Tehran, Iran. He is currently Assistant Professor of Chemical Engineering at University of Tabriz, Tabriz, Iran. His research interests are molecular simulation of nanoscale phenomena. Also, he works on interfacial phenomena, membrane-based water desalination, and gas separation systems. His published articles are related to the subjects including gas hydrates, nanofluids, nanocomposites, water desalination, and drug delivery systems. 